DR. MIRKO MANCHIA (Orcid ID : 0000-0003-4175-6413)

DR. JANUSZ RYBAKOWSKI (Orcid ID : 0000-0003-0577-0381)

DR. LARS KESSING (Orcid ID : 0000-0001-9377-9436)

DR. MARTIN ALDA (Orcid ID : 0000-0001-9544-3944)

DR. LEONARDO TONDO (Orcid ID : 0000-0002-1624-0522)

Article type : Commentary

\title{
Lithium and bipolar depression
}

Mirko Manchia ${ }^{1,2}$, Janusz K. Rybakowski ${ }^{3}$, Gabriele Sani ${ }^{4,5,6}$, Lars V. Kessing, ${ }^{7}$ Andrea Murru, ${ }^{8}$ Martin Alda ${ }^{9 *}$, Leonardo Tondo, ${ }^{6,10^{*}}$

${ }^{1}$ Section of Psychiatry, Department of Medical Sciences and Public Health, University of Cagliari, Cagliari, Italy

${ }^{2}$ Department of Pharmacology, Dalhousie University, Halifax, Nova Scotia, Canada

${ }^{3}$ Department of Adult Psychiatry, Poznan University of Medical Sciences, Poznan Poland

${ }^{4}$ NeSMOS Department, Sapienza University, Roma

${ }^{5}$ Psychiatric Department, Sant'Andrea Hospital, Roma

${ }^{6}$ Lucio Bini Mood Disorder Centers, Cagliari and Rome, Italy

${ }^{7}$ Psychiatric Center Copenhagen, Rigshospitalet, University of Copenhagen, Denmark.

${ }^{8}$ Barcelona Bipolar and Depressive Disorders Unit, Institute of Neuroscience, Hospital

Clinic, University of Barcelona, IDIBAPS, CIBERSAM, Barcelona, Catalonia, Spain.

${ }^{9}$ Department of Psychiatry, Dalhousie University, Halifax, Nova Scotia, Canada

${ }^{10}$ McLean Hospital-Harvard Medical School, Boston, MA, USA.

This article has been accepted for publication and undergone full peer review but has not been through the copyediting, typesetting, pagination and proofreading process, which may lead to differences between this version and the Version of Record. Please cite this article as doi: 10.1111/bdi.12781

This article is protected by copyright. All rights reserved. 
* These authors contributed equally

Correspondence:

Mirko Manchia, MD, PhD

Section of Psychiatry, Department of Medical Sciences and Public Health

University of Cagliari, Via Liguria, 19 - 09127 Cagliari, Italy

Tel. +393404189755

E-mail address: mirkomanchia@unica.it; Mirko.Manchia@dal.ca (M. Manchia)

Running title: Lithium in bipolar depression

Kelly ${ }^{1}$ has recently disputed the recommendations of several international guidelines on the use of lithium in bipolar depression. In his scrutiny, the author points to three main errors that seem to have affected systematically ten international guidelines, namely the Woozle effect (evidence by citation), reference inflation (inappropriate citation of pivotal, generally old, studies) and belief perseverance (inability to modify evidence-base recommendations despite the presence of contrary data). We concur with the author that the evidence supporting the effectiveness of lithium in acute bipolar depression, and to a lesser degree also in major depressive episodes, remains inadequate. ${ }^{2,3}$ A different matter is, in our opinion, to label guidelines recommendations as inaccurate or biased, even if, as the author stated, no deceptive intentions were present. As Kelly points out, lithium might have been the most studied drug in bipolar disorder, but its effect in acute bipolar depression remains largely untested. However, some comments could be made: 1) The author claims that these phenomena interfere with our understanding of the scarce evidence, but no guideline misinterpreted data on the efficacy of lithium in bipolar depression. Rather, panels of experts 
have tried to formulate recommendations placing the available evidence, even if limited, into a real-world clinical context. Illustrative of this is the explanation formulated in the Canadian Network for Mood and Anxiety Treatments (CANMAT) guideline concerning the possible influence of low serum lithium levels on the scarce therapeutic efficacy in the only large double blind placebo controlled trial conducted to date in bipolar depression. ${ }^{4}$ 2) We need to differentiate between evidence and recommendation. This is especially relevant in a situation where there are no easy alternatives. Even if aware of the weak evidence for the acute antidepressant effect of lithium, experts panels might recommend it as first-line treatment for bipolar disorder in depressive phases for its effect in preventing further manic, hypomanic, or depressive recurrences. 3) The well-demonstrated antisuicidal effects of lithium justify its use in acute depressive episodes, even in non-bipolar disorder subjects. 4) Lithium augmentation of antidepressants in treatment-resistant depressive episodes has been established as an effective therapeutic option. ${ }^{5}$ This effect may be associated with the presence of mixed features in treatment-resistant depressions. 5) Rather than indicating a methodological problem, the apparent inconsistencies found in the guidelines by Kelly highlight the absence of valid alternative treatments for bipolar disorder.

In conclusion, we reckon that the guideline recommendations for using lithium in bipolar depression should not be chosen as case in point of unintentional, biased, assessment of the literature. On the contrary, they emphasize the surprisingly large gap in the knowledge on the effectiveness of lithium in depressive phases despite the extensive use of this mood stabilizer since the 1950s and call for more clinical and basic research in this relevant area.

This article is protected by copyright. All rights reserved. 


\section{References}

1. Kelly T. Lithium and the Woozle effect. Bipolar Disord. February 2019.

2. Vázquez GH, Holtzman JN, Tondo L, Baldessarini RJ. Efficacy and tolerability of treatments for bipolar depression. J Affect Disord. 2015;183:258-262.

3. Undurraga J, Sim K, Tondo L, et al. Lithium treatment for unipolar major depressive disorder: Systematic review. J Psychopharmacol. 2019;33(2):167-176.

4. Yatham LN, Kennedy SH, Parikh S V., et al. Canadian Network for Mood and Anxiety Treatments (CANMAT) and International Society for Bipolar Disorders (ISBD) 2018 guidelines for the management of patients with bipolar disorder. Bipolar Disord. 2018;20(2):97-170.

5. Crossley NA, Bauer M. Acceleration and augmentation of antidepressants with lithium for depressive disorders: two meta-analyses of randomized, placebo-controlled trials. $J$ Clin Psychiatry. 2007;68(6):935-940.

This article is protected by copyright. All rights reserved. 